\title{
Heuristic for Multi-objective Solution of the Periodic Vehicle Routing Problem
}

\author{
Angélica Tenahua ${ }^{1}$, Elías Olivares-Benitez ${ }^{1}$, Jorge Esparza ${ }^{2}$ \\ ${ }^{1}$ Universidad Popular Autónoma del Estado de Puebla, \\ Mexico \\ ${ }^{2}$ Universidad Anáhuac, Puebla, \\ Mexico \\ \{angelicamaria.tenahua, elias.olivares\}@upaep.mx, jorge.esparza@anahuac.mx
}

\begin{abstract}
This paper presents a methodology for solving the Periodic Vehicle Routing Problem (PVRP). Customer locations each with a certain daily demand are known, as well as the capacity of vehicles. In this work, the problem is solved by means of a two-phase heuristic. The first phase is the itinerary assignment to visit each customer, and in the second phase the routing is done for each day of the itinerary. The itinerary assignment is made randomly and the routing is solved using the Savings Heuristic. The problem is treated as a bi-objective problem that minimizes the total distance travelled in the time horizon, minimizing also the maximum number of routes in any day. Three instances were solved identifying the non-dominated solutions and presenting them in a graph of the Pareto front. Computational results show that the proposed approach can yield good quality solutions within reasonable running times.
\end{abstract}

Keywords: Vehicle routing problem, non-dominated solutions, multi-objective, savings heuristic.

\section{Introduction}

The Vehicle Routing Problem (VRP) is a classic combinatorial optimization problem, widely studied. The first publications were in 1959 [1], and the problem is to generate routes at minimal cost, for a given set of vehicles with homogeneous capacity [2]. In order to include features to the real world, variants of the VRP have been studied. Some of them are, vehicles with heterogeneous fleet, time windows, periodical visits, more than one depots, etc. The main variants of the VRP can be found in publication [3]. The PVRP (Periodic Vehicle Routing Problem) can be considered as a generalization of the conventional VRP, which looks for determining an optimal set of daily routes for a planning horizon of time. Customers should be visited on different days during the planning horizon. According to the number of visits required by the customer, there are combinations of these visits, named itineraries. The solution of the PVRP implies solving two entangled problems: an assignment problem and the vehicle routing problem. In the assignment problem it is necessary to determine a valid itinerary (visiting days) for each customer within the planning horizon. The other is the vehicle 
routing problem for each day. The PVRP is considered a NP-hard problem [4], so the techniques with more efficient solution for these problems are the heuristics. The most common heuristics used for this problem are: Tabu Search [5, 6], GRASP [7], Ant Colony Optimization (ACO) [8], Variable Neighbourhood Search (VNS) [9] and a hybrid heuristic based on joint coverage [10].

The total cost to minimize includes the costs associated with the distances travelled, vehicle capacity, transit time, fuel, etc. In this paper the distance is directly related to the cost. For more information about costs, the reader can review the publication in [11] that made a review of fixed and variable costs of transportation. In this work, we are interested in giving solutions to problems that may happen in real situations for the companies. This paper considers the transports (delivery trucks) with the same capacity, known as homogeneous fleet. The planning horizon is of six days, which may result in a week from Monday to Saturday. The solution of PVRP in this work is made first with assignment of itinerary to each customer based on number of visits required during the planning horizon. After that routing is performed by the Savings Method [12].

The paper is structured as follows. Section 2 is the Literature Review where the major papers have served as a reference and analysis for this study. Problem description is made in Section 3. The development of the methodology is shown in Section 4, which states how the PVRP results are obtained. Section 5 describes the implementation details of the method proposed. The results obtained are shown in Section 6. Finally in Section 7 the conclusions of this work are drawn.

\section{Literature Review}

This section presents the literature review of the papers that have served as a reference and analysis of this study. Each of them is mentioned in a very general way presenting the problem, the solution method and the results obtained.

Mendez et al. [13] solved the infectious waste collection problem as a Periodic Vehicle Routing Problem, using a hybrid technique that involves an evolutionary algorithm for optimal planning of the collection days, and a local search algorithm to determine the set of optimal routes every day. They divide the problem in the same way as we propose since it is natural to construct that hierarchy. Gaudioso and Paletta [14] describe a model for the optimal management of a commodity. The goal is to schedule the deliveries according to feasible combinations of delivery days and to determine the scheduling and routing policies over the planning horizon for a maximum number of vehicles simultaneously employed, i.e., the fleet size. Heuristic algorithms were proposed: An implicit enumeration algorithm for the feasible combinations of delivery to each customer, a heuristic algorithm for assignment of customers to routes, and Bin Packing algorithm for scheduling of routes. In this paper, the fleet size is unknown, but the objective is to get the minimum. Wen et al. [15] consider the Dynamic Multi-Period Vehicle Routing Problem, which deals with the distribution of orders from a depot to a set of customers over a multi-period time horizon. Customer orders and their feasible service periods are dynamically revealed over time. The objectives were to minimize total travel costs and customer waiting, and to balance the daily workload over the planning horizon. This problem originates from a large distributor operating in Sweden. It is modelled as a mixed integer linear program, and solved by means of a three-phase 
heuristic that works over a rolling planning horizon. The multi-objective aspect of the problem is handled through a scalar technique approach. Computational results show that the proposed approach can yield high quality solutions within reasonable running times. In this paper although the model is multi-objective, demands and visit requirements are fixed and not dynamic as in the work of Wen et al. (2009), which is a more general problem, however, has served as a guide considering fixed visits.

Francis and Smilowitz [16] presented a continuous approximation model for the Period Vehicle Routing Problem with Service Choice (PVRP-SC). The PVRP-SC is a variant of the Period Vehicle Routing Problem in which the visit frequency to nodes is a decision of the model. This variation can result in more efficient vehicle tours and/or greater service benefit to customers. The continuous approximation model can facilitate strategic and tactical planning of periodic distribution systems and evaluate the value of service choice. Francis and Smilowitz suggested making a parametric analysis of future conditions, which may be useful to test the sensitivity of the solutions for future scenarios before committing to the highest service levels. For this work, no time windows are considered, but the mathematical model from Smilowitz and Francis [17] proposed, is a great help in this paper considering large windows of time. Griffis and Bell [18] made a comparison between results obtained from five different instances of the type: random, cluster, Sector, Rural and Urban Coastal, through the metaheuristic Ant Colony Optimization (ACO), and Clark and Wright heuristic. The best results are Sector type. For future work related to this article would make an interesting analysis with the five types of population distribution and identify if any of them has better solutions. Ballou and Agarwal [19] made a comparison between the methods of Savings, Cluster and sweep under five types of population distribution, random, cluster, Sector, Urban-rural and Coastal. The results reflect an advantage of the Savings method vs. Cluster and sweep methods with different types of distribution. In this paper, savings method is used to find routes each day of the period.

\section{Problem Description}

Consider the graph $G=(N, A)$ and period $n p$ days (planning horizon), where $N$ is the set of nodes representing customers and $A$ is the set of arcs connecting the nodes. Each customer requires the collection task with a frequency $f_{i}$ measuring the number of times the customer $i$ must be visited with $1 \leq f_{i} \leq n p$. A combination of visit times (days) into the planning horizon is named an itinerary. The basic PVRP is to select an itinerary for node $i$ and solve a vehicle routing problem for each day of the selected itineraries, in order to minimize the total cost of travel. As mentioned previously, it is a NP-hard problem because it includes the VRP with single period as a special case [13].

Christofides and Beasley [20] proposed the PVRP model as follows. Let $S_{i}$ be the set of allowable itineraries for customer $i$. Let $x_{i k}=1$ if the $k$ th itinerary is chosen for $i, 0$ otherwise. Let $n$ be the total number of customers and $T$ the number of days in the period. Let $a_{k t}=1$ if day $t$ is in itinerary $k, 0$ otherwise; $q_{i}$ be the demand of customer $i$ (for each delivery); $c_{i j}$ be the travel time from customer $i$ to customer $j ; N=(i \mid i=1$, $2, \ldots, n)$ be the set of customers; $Q$, be the capacity of vehicle $r ; D_{r}$ be the total allowable driving time of vehicle $r$; and $R_{t}$ be the given set of available vehicles for day $t$. Let $v_{i t}=1$ if customer $i$ is visited on day $t, 0$ otherwise; with the depot 
Angélica Tenahua, Elías Olivares-Benitez, Jorge Esparza

represented by customer 0 define $\mathrm{v}_{0 t}=1, t=1, \ldots, T$, and let $u_{i j t r}=1$ if vehicle $r \in R_{t}$ goes from $i$ to $j$ on day $t, 0$ otherwise; then the program is to

Minimize

$$
\sum_{t=1}^{T} \sum_{i=0}^{n} \sum_{j=0}^{n} \sum_{r \in R_{t}} c_{i j} u_{i j t r}
$$

Such that

$$
\begin{aligned}
& \sum_{\mathrm{k} \in \mathrm{S}_{\mathrm{i}}} \mathrm{x}_{\mathrm{ik}}=1, \forall \mathrm{i}(\mathrm{i} \neq 0) \\
& \mathrm{v}_{\mathrm{it}}=\sum_{\mathrm{k} \in \mathrm{S}_{\mathrm{i}}} \mathrm{x}_{\mathrm{ik}_{\mathrm{k}}} \mathrm{a}_{\mathrm{kt}}, \forall \mathrm{t}, \forall \mathrm{i}(\mathrm{i} \neq 0) \\
& \sum_{r \in R_{t}} u_{i j t r} \leq \frac{v_{i t}+v_{j t}}{2}, \forall i, j, t(i \neq j) \\
& \sum_{i=0}^{n} u_{i p t r}=\sum_{j=0}^{n} u_{p j t r}, \forall p, t, r \in R_{t}, \\
& \sum_{\mathrm{r} \in \mathrm{R}_{\mathrm{t}}} \sum_{\mathrm{i}=0}^{\mathrm{n}} \mathrm{u}_{\mathrm{ijtr}}=\mathrm{v}_{\mathrm{jt}}, \forall \mathrm{j}, \mathrm{t}(\neq 0) \\
& =\left|\mathrm{R}_{\mathrm{t}}\right| \forall \mathrm{t}(\mathrm{j}=0) \\
& \sum_{i \in W} \sum_{j \in W}^{n} u_{i j t r}<|W|-1, \forall t, r \in R_{t}, \forall W \subseteq N \\
& \sum_{j=1}^{n} u_{0 j t r}<1, \forall t, r \in R_{t} \\
& \sum_{i=1}^{n} q_{i}\left(\sum_{j=0}^{n} u_{i j t r}\right)<Q_{r}, \forall t, r \in R_{t} \\
& \mathrm{x}_{\mathrm{ik}} \in(0,1), \forall \mathrm{i}, \mathrm{k} \in \mathrm{S}_{\mathrm{i}} \\
& \mathrm{u}_{\mathrm{ijtr}} \in(0,1), \forall \mathrm{i}, \mathrm{j}, \mathrm{t}, \mathrm{r} \in \mathrm{R}_{\mathrm{t}} \text {. }
\end{aligned}
$$


Eq. (1) is the objective function to evaluate the total travel cost. Eq. (2) ensures that only one delivery combination is chosen for each customer, Eq. (3) ensures that a customer is only visited on a particular day if the delivery combination chosen has a delivery on that day, Eq. (4) ensures that no vehicle can go between two customers on a particular day unless they are both scheduled for delivery on that day, Eq. (5) ensures that if a vehicle visits a customer it also leaves that customer, Eq. (6) ensures that each customer is visited on the days that it is scheduled for delivery. Eq. (7) is the set of subtour elimination constraints. Eq. (8) ensures that a vehicle can only be used at most once. Eq. (9) is the vehicle capacity constraint.

\section{Methodology}

It is considered a planning horizon of six days, so $n p=6$. Each customer has an associated frequency of visits $f_{i}$. It is possible to represent itineraries in Table 1 . If a customer is visited on day $t$, the value is 1,0 otherwise. The pattern of visits can be represented by a set $P$ of binary vectors $x$. Each element has a value of 1 if the customer is visited on day $t, 0$ otherwise. Some restrictions are implemented to prevent consecutive visits. Assignment of itineraries is random according between all possible itineraries that meet $f_{i}$ of each customer $i$. Figure 1 presents graphically the methodology explained.

Table 1. Itineraries for one, two or three visits.

\begin{tabular}{|c|c|c|c|c|c|c|c|}
\hline Itinerary & Number of visits & Day 1 & Day 2 & Day 3 & Day 4 & Day 5 & Day 6 \\
\hline 1 & 1 & 1 & 0 & 0 & 0 & 0 & 0 \\
\hline 2 & 1 & 0 & 1 & 0 & 0 & 0 & 0 \\
\hline \multicolumn{8}{|l|}{$\ldots$} \\
\hline 7 & 2 & 1 & 0 & 1 & 0 & 0 & 0 \\
\hline 8 & 2 & 1 & 0 & 0 & 1 & 0 & 0 \\
\hline \multicolumn{8}{|l|}{$\cdots$} \\
\hline 16 & 3 & 1 & 1 & 0 & 1 & 0 & 0 \\
\hline 17 & 3 & 1 & 1 & 0 & 0 & 1 & 0 \\
\hline$\ldots$ & & & & & & & \\
\hline
\end{tabular}

Table 2 presents an example of generated routes each day, for a planning horizon of 6 days. For example observe customers 4, 14 and 9 they require to be visited 1, 2 and 3 times a week respectively. For customer 4 , the set $P$ is $P=\{100000,010000,001000$, $000100,000010,000001\}$. The element of the set that was assigned is the last element, which means customer 4 is visited on day 6 of the planning horizon. For customer 14, it is visited on day 3 and 5 of the period. Customer 9 is visited on days 1, 4 and 6 . 


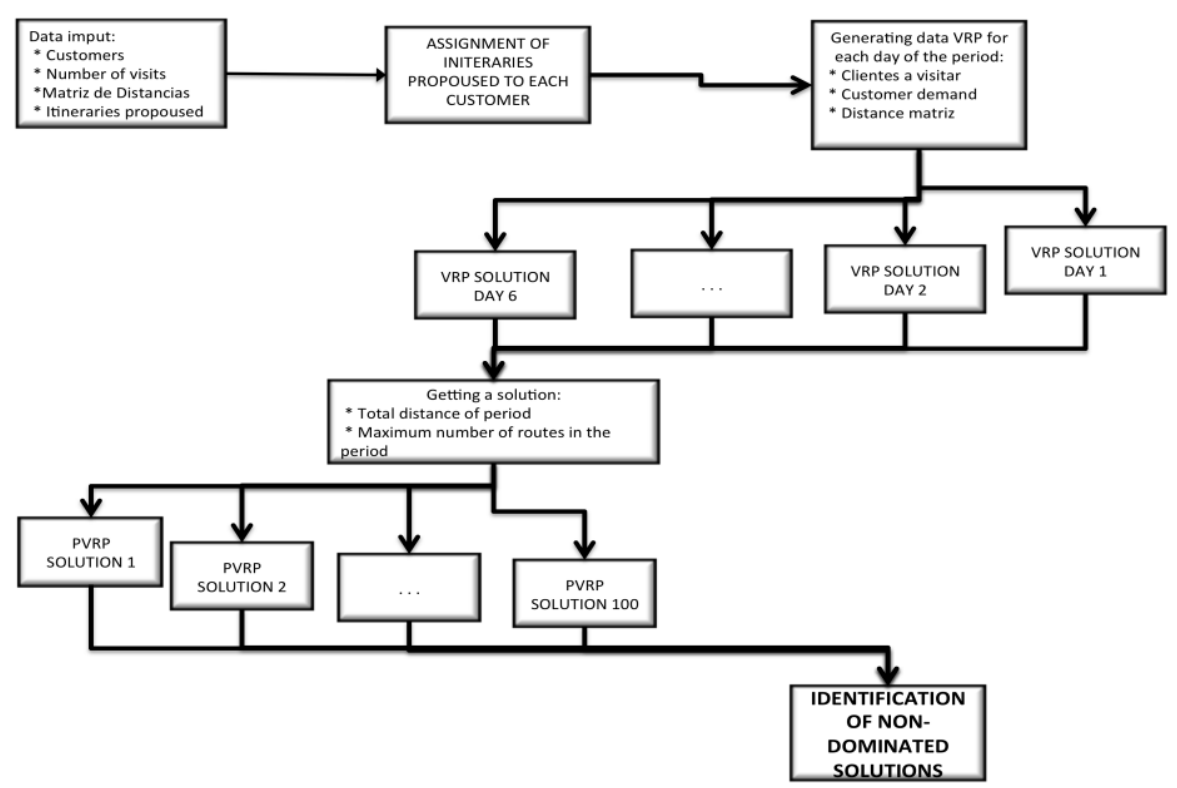

Fig. 1. Methodology diagram.

Table 2. Example of routes generated on each day of a planning horizon.

\begin{tabular}{cl}
\hline Day & Sequences of nodes in the routes for each day \\
\hline 1 & 09151003120 \\
2 & 026508120 \\
3 & 0156140 \\
4 & 01515091103120 \\
5 & 0821403130 \\
6 & 067904110 \\
\hline
\end{tabular}

\section{Details of Implementation}

The algorithm was coded in $\mathrm{C}++$ programming language to solve three instances. Details of instances are presented in Table 3. The computer is a Mac running OS $\mathrm{X}$ Processor 2.4 GHz Intel Core 2 Duo and Memory 5 GB 1067 MHz DDR3. Average runtime was 2725 seconds.

Table 3. Data of the instances worked.

\begin{tabular}{cccc}
\hline Instance & Total Customers & Average demand & Stantandar deviation of demand \\
\hline 1 & 15 & 389.33 & 54.95 \\
2 & 15 & 296.13 & 174.17 \\
3 & 25 & 2.72 & 1.21 \\
\hline
\end{tabular}


According to the number of visits required by each costumer, an itinerary is assigned randomly. Once every customer has an itinerary assigned, the total costumers to be visited each day is known. With the last information it is possible to generate a distance matrix for each day of the period, for routing.

The nodes to visit each day on the planning horizon, is solved as a VRP. The Savings Method obtains the solutions of VRP. The solution on a planning horizon has the following information: the number of nodes to be visited every day on the planning horizon, the nodes to be visited every day of the planning horizon, the number of routes that are generated each day, routes, the distance travelled on each route, the distance travelled each day on the period, and the total distance travelled throughout the period. To obtain solutions for different assignments of itineraries, one hundred solutions are created and evaluated for each instance. Subsequently the non-dominated solutions are identified to construct an approximate Pareto front. To define non-dominated solutions in this paper, we consider two objective functions: the total distance and the maximum number of routes between all days in a period. An example of solution of a period is presented in Table 2, in which the routes are indicated in each day. Note day 1 , in which two routes were generated. The routes are separated by node 0 which is the depot. In the same example, it can be observed that on day 3 only one route is generated, while on day 4 , three routes are generated.

\section{Results}

Outcomes of interest are the total distance and the maximum number of routes during the period. Table 4 presents the results and shows the non-dominated solutions of the three instances with average execution time of 2725 seconds. Figure 3 is the graph of the solution with instance 1 , identifying with dark and bigger square the non-dominated solution.

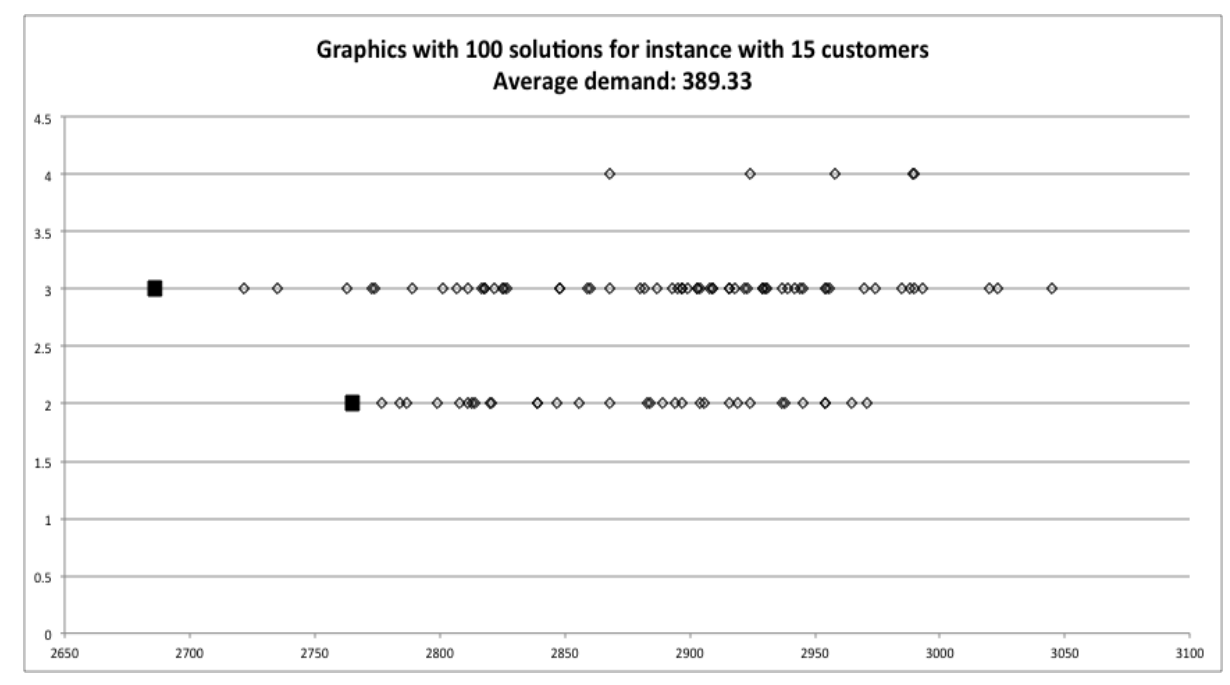

Fig. 3. Shows the graphic with 100 solutions of the PVRP for Instance 1. 
Angélica Tenahua, Elías Olivares-Benitez, Jorge Esparza

Table 4. Non-dominated solutions from three instances.

\begin{tabular}{cccc}
\hline Instance & Number of solution & $\begin{array}{c}\text { Total distance travelled during } \\
\text { the period }\end{array}$ & $\begin{array}{c}\text { Maximum number of routes } \\
\text { during the period }\end{array}$ \\
\hline 1 & 12 & 2686.00 & 3 \\
1 & 99 & 2765.00 & 2 \\
2 & 82 & 3295.00 & 2 \\
3 & 55 & 130.04 & 3 \\
3 & 79 & 132.01 & 2 \\
\hline
\end{tabular}

\section{Conclusions}

One hundred solutions for each instance, allow finding one or two non-dominated solutions. It indicates that there is a high correlation between distance and maximum number of routes per period. The assignment is random so two analyses are proposed to address this for future works, the first is in which the assignation has certain criteria that could improve and verify the solutions proposed improvement. The second proposal is to do intra-route and inter- routes changes. A further opportunity is to find another trade- off to identify non-dominated solutions.

\section{References}

1. Dantzig, G., Ramser, J.: The Truck dispatching problem. Management Science, Vol. 6, No. 1, pp. 80-91 (1959)

2. Abreu, R., Arroyo, J.: Aplicação da heurística ILS para o Problema de Roteamento de Veículos Periódico com Frota Heterogênea e Custos Fixos (2014)

3. Toth, P., Vigo, D.: The Vehicle Routing Problem (2014)

4. Chao, I., Golden, B., Wasil, E.: An improved heuristic for the period vehicle routing problem. Networks, Vol. 26, Issue 1, pp. 25-44 (1995)

5. Cordeau, J., Gendreau, M., Laporte, G.: A tabu search heuristic for period and multi-depot vehicle routing problems. Networks, Vol. 30, pp. 105-119 (1997)

6. Cordeau, J., Laporte, G., Mercier, A.: A unified tabu search heuristic for vehicle routing problems with time windows. Journal of the Operational research society, Vol. 52, No. 8, pp. 928-936 (2001)

7. Pacheco, J., Álvarez, A., García, I., Angel-Bello, F.: Optimizing vehicle routes in a bakery company allowing flexibility in delivery dates. Journal of the Operational Research Society, Vol. 63, No. 5, pp. 569-581 (2012)

8. Bell, J., Griffis, S.: Swarm intelligence: Aplication of the ant colony optimization algorithm to logistics-oriented vehicle routing problems. Journal of Business Logistics, Vol. 31, No. 2, pp. 157-175 (2010)

9. Hemmelmayr, V., Doerner, K., Harlt, R.: A variable neighborhood search heuristic for periodic routing problems. European Journal of Operational Research, Vol. 195, No. 3, pp. 791-802 (2009)

10. Cacchiani, V., Hemmelmayr, V., Tricoire, F.: A set-covering based heuristic algorithm for a periodic vehicle routing problem. Discrete Applied Mathematics, Vol. 163, Parte 1, pp. 53-64 (2014)

11. Da Silva, I., Reis, R., Gomes, M.: Custos e otimização de rotas no transporte de leite a latão e a granel: um estudo de caso. Organizações Rurais \& Agroindustriais, Vol. 2, No. 1 (2011) 
12. Clarke, G. Wright, J.: Scheduling of Vehicles from a Central Depot to a Number of Delivery Points. Operations Research, Vol. 12, Issue 4, pp. 568-581 (1964)

13. Méndez, A., Pontin, M., Ziletti, M., Chávez, L.: Heurísticas para la resolución de un Problema de Ruteo de Vehículos Periódico. Mecánica Computacional, Vol. 24, pp. 29512960 (2005)

14. Gaudioso, M., Paletta, G.: A Heuristic for the Periodic Vehicle Routing Problem. Transportation Science, Vol. 26, No. 2, pp. 86-92 (1992)

15. Wen, M., Cordeau, J.F., Laporte, G., Larsen, J.: The Dynamic Multi-Period Vehicle Routing Problem. Computers \& Operations Research, Vol. 37, Issue 9, pp. 1615-1623 (2010)

16. Francis, P., Smilowitz, K.: Modeling Techniques for Periodic Vehicle Routing Problems. Transportation Research Part B Methodological, Vol. 40, Issue 10, pp. 872-884 (2006)

17. Christofides, N., Beasley, J.E.: The Period Routing Problem. Networks, Vol. 14, Issue 2, pp. 237-256 (1984) 